\title{
Técnica Artroscópica Del Pinzamiento Ulnocarpiano
}

\section{Arthroscopic Technique for Ulnocarpal Impingement}

\author{
Francisco Javier Lucas ${ }^{1}$ Eva Guisasola ${ }^{1}$ Rogelio Carratalá ${ }^{1,2}$ Ignacio Miranda ${ }^{3(D)}$ \\ 1 Unidad de cirugía de mano y miembro superior, Hospital Quiron \\ Salud Valencia, Valencia, Spain \\ 2 Mutua laboral ASEPEYO, Hospital Intermutual de Levante,

\begin{abstract}
Address for correspondence Francisco Javier Lucas García, Unidad de cirugía de mano y miembro superior, Hospital Quiron Salud Valencia, Valencia, Spain (e-mail: drlucas@cirugiamanovalencia.com).
\end{abstract} Valencia, Spain

${ }^{3}$ Hospital Universitario La Fe, Valencia, Spain

Rev Iberam Cir Mano 2019;47:141-146.

\author{
Resumen \\ Palabras clave \\ - pinzamiento \\ - HALT \\ - oblea
}

El pinzamiento ulnocarpiano es una de las causas más frecuentes de dolor en el borde ulnar de la muñeca. Se trata de una patología degenerativa producida por la impactación crónica del cúbito contra el fibrocartílago triangular y la parte ulnar del carpo. La evolución natural de esa enfermedad es progresiva y en diferentes etapas. La técnica quirúrgica consiste en determinar en qué etapa nos encontramos e ir añadiendo gestos quirúrgicos en función de la etapa siendo el objetivo del tratamiento quirúrgico realizar una descompresión de la columna ulnar y reparar las lesiones asociadas. Presentamos la técnica quirúrgica que se realiza desde la fase más inicial hasta la afectación de toda la columna ulnar.

Ulnocarpal impingement is one of the most frequent causes of pain in the ulnar edge of the wrist. It is a degenerative pathology produced by the chronic ulna impactation against triangular fibrocartilage and the ulnar carpus side. Its usual evolution is produced in progressive stages. The surgical technique consists in determining the stage and perform proper surgical procedures. The objective of the surgical treatment will be to perform a decompression of the ulnar side and repair all associated lesions. We present the surgical technique that is performed from the initial stage to the total ulnar side affectation.

\section{Introducción}

El pinzamiento ulnocarpiano es una de las causas más frecuentes de dolor en el borde ulnar de la muñeca junto con las lesiones del complejo del fibrocartílago triangular (FCT) y las lesiones del ligamento lunopiramidal. ${ }^{1}$ Se trata de una patología degenerativa y progresiva producida por la impactación crónica del cúbito contra el fibrocartílago triangular (FCT) y la parte ulnar del carpo. La causa más frecuente es secundaria a fracturas de radio distal, debido a la inversión del índice radioulnar distal por el acortamiento relativo del radio.

received

September 26, 2019

accepted

October 3, 2019
DOI https://doi.org/

10.1055/s-0039-3399516. ISSN 1698-8396.
La evolución usual de esta enfermedad (- Fig. 1) es progresiva $y$ en diferentes etapas. Se inicia con un debilitamiento central que puede conducir a la perforación central del FCT, lesión condral con pérdida completa de cartílago en el borde proximal-ulnar del semilunar y proximal-radial del hueso piramidal, lesión del ligamento lunopiramidal y finalmente lesión condral del polo proximal del ganchoso o síndrome HALT (Hamate Arthritis Lunotriquetral Ligament Tears). ${ }^{2}$ Esa secuencia sigue la clasificación artroscópica de las lesiones del complejo del FCT tipo II propuesta por Palmer. ${ }^{3}$
Copyright $\odot 2019$ Thieme Revinter Publicações Ltda, Rio de Janeiro, Brazil
License terms

(ㄷ) (i) $\ominus$ (\$) 
El objetivo del tratamiento quirúrgico será realizar una descompresión de la columna ulnar a nivel de la articulación ulnocarpiana y reparar las lesiones asociadas a la impactación ulnar como lesiones del FCT, lesiones condrales en el semilunar, piramidal y cabeza ulnar, lesiones del ligamento lunopiramidal, sinovitis, inestabilidades del carpo y artrosis articular, ${ }^{4}$ permitiendo un tratamiento más completo y con unos resultados muy satisfactorios.

\section{Indicaciones y Contraindicaciones}

Las indicaciones de la técnica quirúrgica artroscópica para el pinzamiento ulnocarpiano son las mismas que las utilizadas en cirugía abierta y abarcan todas las fases del pinzamiento ulnocarpiano desde la perforación central del FCT, lesiones condrales en la cabeza ulnar, polo proximal de semilunar y piramidal, lesión del ligamento lunopiramidal y síndrome HALT. El hallazgo de una artrosis extensa en la articulación radiocubital distal (ARCD), radiocapiana o mediocarpiana así como lesiones no reparables del CFCT que produzcan inestabilidad de la ARCD contraindicarían la realización de la siguiente técnica, además, como contraindicaciones relativas, se incluirían cualquier circunstancia que desaconsejen la realización de una artroscopia de muñeca como infecciones, síndrome de dolor regional complejo...



Figura 1 (A) Dibujo de lesiones que pueden aparecer en el síndrome de impactación ulnar. Perforación central del FCT, lesiones condrales en el semilunar, el piramidal y la cabeza ulnar y rotura del ligamento lunopiramidal. (B) Imagen artroscópica desde el portal 3-4; donde se aprecia lesión extensa del cartílago en el semilunar (SL) y una rotura central del FCT con visión directa de la cabeza ulnar (U). (C) Radiografía AP de muñeca mostrando un cúbito plus. (D) Imagen de resonancia magnética (IRM) que muestra el edema óseo a nivel del polo proximal del hueso semilunar por pinzamiento ulnocarpiano.

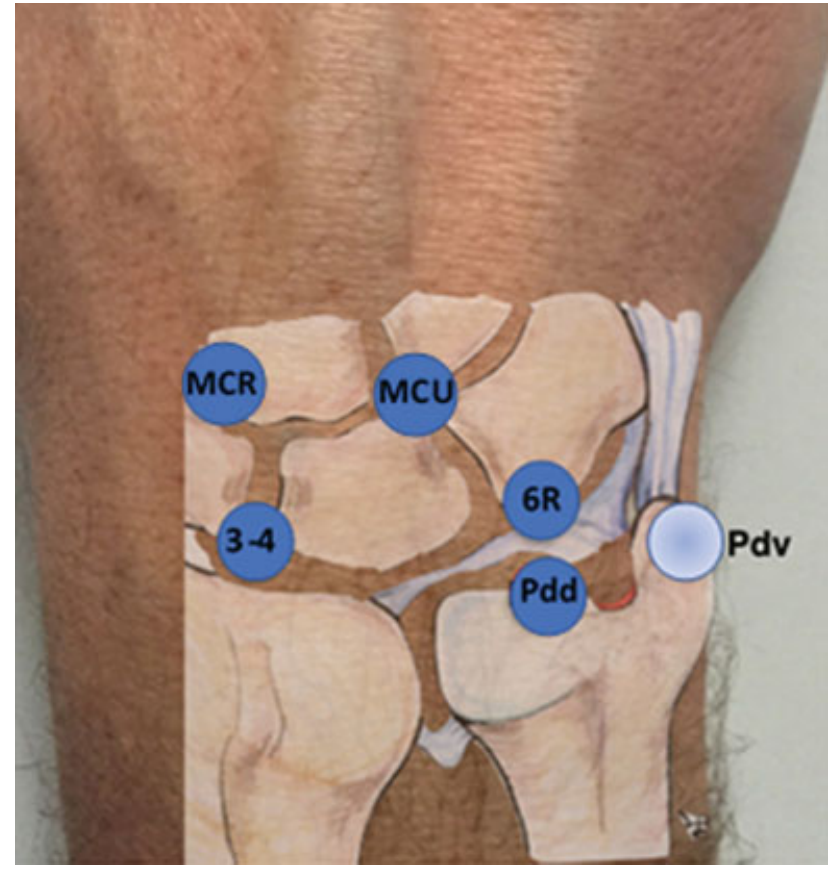

Figura 2 Imagen que muestra los portales radiocarpianos 3-4 y 6R, los portales mediocarpianos MCR (mediocarpiano radial) y MCU (mediocarpiano ulnar) y de la articulación radiocubital distal Pdd (Portal dorsal) y Pdv (Portal volar).

Técnica Artroscópica DEL Pinzamiento Ulnocarpiano La artroscopia de muñeca es la mejor herramienta para evaluar la gravedad de los pinzamientos ulnocarpianos ya que proporciona una visión directa de las lesiones tanto condrales como del FCT y ligamentos interóseos.

\section{Posicionamiento y Portales}

Se utiliza una distribución estándar para una artroscopia de muñeca situando al paciente en decúbito supino con el brazo sobre una mesa de mano con manguito de isquemia en el brazo a nivel proximal y bajo anestesia locorregional. El codo se coloca a $90^{\circ}$ flexión y el antebrazo bajo tracción axial utilizando una torre de artroscopia y trampas de dedos en el $2^{\circ}$ y 3 er radios.

Los portales empleados en esa técnica son (-Fig. 2):

1. A nivel radiocarpiano los portales $3-4$, que será el portal principal de visión y los portales 4-5 o 6R como portales de trabajo. El 6R permite un mejor manejo de los instrumentos al estar más alejados de la óptica. Además, se localiza directamente distal al FCT y la cabeza ulnar.

2. A nivel mediocarpiano se utilizan los portales mediocarpiano ulnar (MCU) y mediocarpiano radial (MCR)

3. A nivel de la articulación radiocubital distal (ARCD) se emplearán el portal dorsal distal de la radiocubital distal (pdd-RCD) y el portal distal volar radioulnar (pdv-RU). ${ }^{5}$ 



Figura 3 (A) Visión artroscópica desde el portal 6R, en la imagen observamos la fosa sigmoidea (FS) del radio. FCT: fibrocartílago triangular; R: radio; U: cabeza ulnar. (B) Visión artroscópica tras completar la resección tipo wafer de la cabeza ulnar. (C) Esquema del procedimiento de wafer artroscópico a través del defecto central del FCT. Con movimientos de pronación y supinación se resecan los márgenes dorsales y volares.
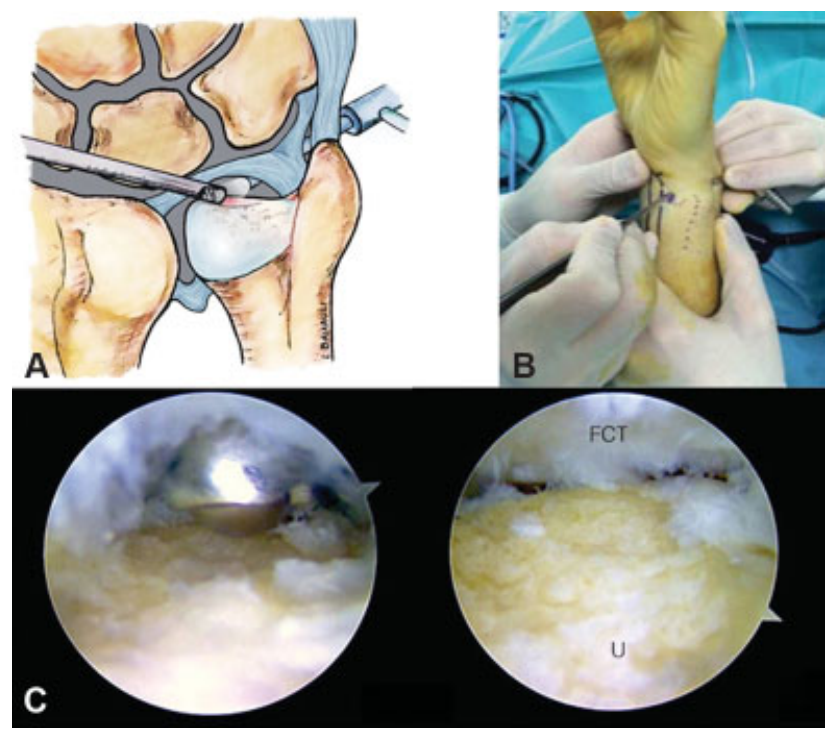

Figura 4 (A) Esquema del procedimiento de wafer artroscópico a través de la articulación radiocubital distal (ARCD) realizado cuando encontramos el fibrocartílago triangular (FCT) intacto. (B) Imagen externa de los portales utilizados durante el procedimiento wafer por los portales de la articulación radiocubital distal. (C) Imagen artroscópica con la óptica en el portal dorsal distal de la ARCD y el sinoviotomo en el portal volar de la ARCD e imagen final tras el procedimiento wafer finalizado con la cabeza ulnar $(U)$ en la parte inferior y el FCT en la parte superior.

\section{Hallazgos Artroscópicos}

Se inicia el procedimiento artroscópico con la óptica a través del portal 3-4 para realizar una exploración completa de la articulación radiocarpiana. Bajo visión directa se realizará el portal 6R o 4-5 como portales de trabajo, según preferencias. Mediante esos portales de trabajo, es posible realizar el
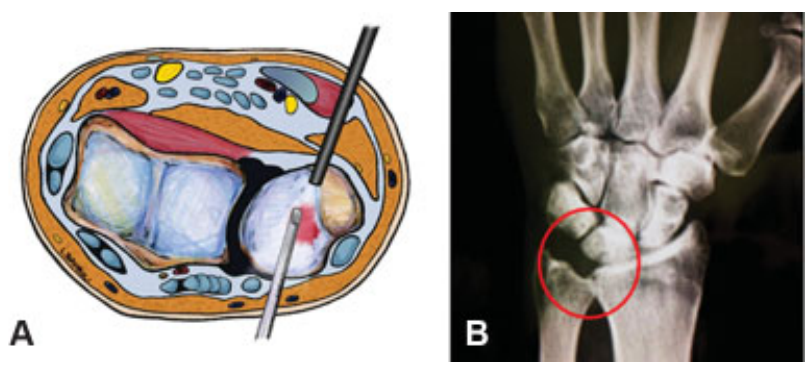

Figura 5 (A) Dibujo de corte transversal de muñeca donde se aprecian las relaciones anatómicas entre las diferentes estructuras y los portales radiocubitales distales. (B) Radiografía anteroposterior de muñeca donde se aprecia la resección de tipo wafer de la cabeza del cúbito.

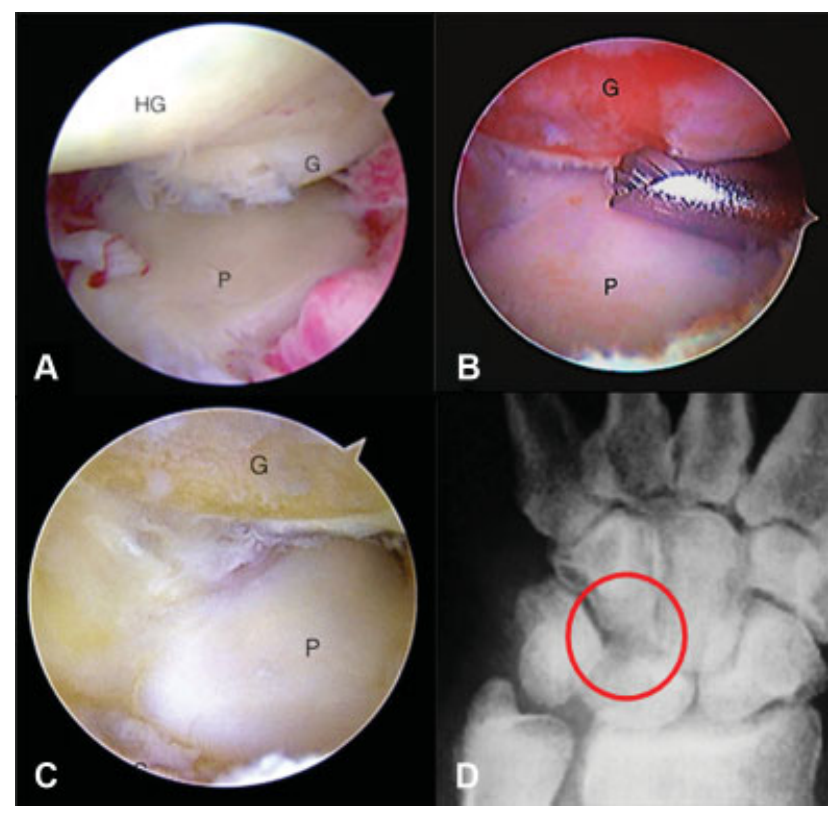

Figura 6 (A) Imagen artroscópica con óptica desde el portal MCR donde se aprecian la superficie distal de piramidal (P), el polo proximal del huego grande (HG) y la lesión condral del polo proximal del hueso ganchoso (G). (B) Imagen artroscópica de la resección del polo proximal del ganchoso con fresa de 2,9mm desde el portal MCU. (C) Imagen artroscópica final de la resección. (D) Radiografía anteroposterior de muñeca donde se aprecia la resección del polo proximal del ganchoso.

tratamiento de las lesiones encontradas y del pinzamiento ulnocarpiano. El instrumental utilizado es un artroscopio de pequeñas articulaciones $(2,5-2,7 \mathrm{~mm})$ y sinoviotomo y fresas de $2,9 \mathrm{~mm}$. La sonda de radiofrecuencia es opcional, ya que se utiliza preferentemente en el tratamiento de las lesiones asociadas.

La metodología más adecuada es realizar una valoración global de las lesiones del borde ulnar, el CFCT, el ligamento lunopiramidal, las superficies articulares de la cabeza ulnar, proximales del semilunar y piramidal a nivel radiocarpiano y proximal del ganchoso a nivel mediocarpiano. El hallazgo más habitualmente encontrado en un pinzamiento ulnocarpiano, es una perforación central del CFCT ( - Figura 3) con la cabeza ulnar sobresaliendo en la articulación radiocarpiana y abundante tejido inflamatorio y fibrótico en el borde ulnar, aunque existen casos con el CFCT íntegro. Se debe evaluar el estado del cartílago en la cabeza ulnar en busca de signos de 
Tabla de TRUCOS Y CONSEJOS

TRUCOS Y CONSEJOS
1. Evitar un fresado excesivo de la cabeza ulnar y del polo proximal del ganchoso utilizando como referencia de profundidad el
grosor de la fresa (aproximadamente $3 \mathrm{~mm}$ ).
2. Realizar una exploración sistemática de las articulaciones radio y mediocarpianas para clasificar de forma adecuada el tipo o
tipos de lesión.
3. Iniciar el tratamiento de proximal a distal, dejando la reparación del ligamento lunopiramidal el último lugar ya que la
inmovilización temporal con AK puede dificultar el resto de los tratamientos.
4. Es preferible la utilización de una fresa ovalada ya que permite una resección más uniforme y es más fácil de controlar.
5. Utilizar irrigación y lavado intermitente mientras se realizan la/las resecciones para eliminar detritus que pueden aumentar la
reacción inflamatoria postquirúrgica.

condropatía, que suelen ser frecuentes, así como lesiones condrales "en espejo" a nivel del polo proximal y ulnar del semilunar y del polo proximal y radial del piramidal.

\section{Técnica Quirúrgica}

Una vez realizado el diagnóstico artroscópico de todas las lesiones, se inicia la limpieza del borde ulnar con la óptica en el portal 3-4 y los instrumentos de trabajo en el portal 6R. En caso de existir una perforación central del FCT y desestructuración de los bordes, debe desbridarse hasta dejar los bordes estables respetando las inserciones periféricas del FCT para no provocar una inestabilidad a nivel de la ARCD. ${ }^{6}$ A través de esa perforación central, se accederá a la ARCD para realizar la resección parcial de la cabeza ulnar. Habitualmente se emplea una fresa de $2,9 \mathrm{~mm}$, resecando entre 2 y $4 \mathrm{~mm}$ de profundidad utilizando el calibre de la fresa (aproximadamente $3 \mathrm{~mm}$ ) como referencia para conseguir una resección adecuada. Se puede realizar un surco de $3 \mathrm{~mm}$ centrado en la cabeza ulnar y completar la resección a nivel volar y dorsal. La utilización de una fresa ovalada permite una resección más uniforme y un mejor control de la misma. La mayor dificultad al realizar la resección por ese método radica en conseguir un acceso óptimo a toda la superficie de la cabeza ulnar a través del defecto central del FCT. La cabeza ulnar no es perfectamente esférica, y por lo tanto es necesario realizar, durante todo el proceso, movimientos de pronación y supinación completa para acometer todos los bordes prominentes de los extremos y resecar las porciones más volares y dorsales de la cabeza ulnar consiguiendo nivelar toda la superficie distal de la cabeza sin afectar la ARCD.

En los casos en los que no presenten un gran defecto en el FCT que permita trabajar cómodamente a través de él, se puede realizar la resección desde el espacio de la ARCD respetando el disco del CFCT (-Figura 4). Para esos casos se emplearán el portal dorsal distal de la radiocubital distal (pdd-RCD) y el portal distal volar radioulnar (pdv-RU) o portal foveal (-Figura 5). La ventaja de esos portales es que proporcionan una visión directa y nivelada de la cabeza ulnar y permiten una resección sin la necesidad de realizar los movimientos de pronosupinación.

Para realizar el procedimiento de resección tipo wafer a través de esos portales, se coloca la óptica en el portal volar y la instrumentación en el dorsal, aunque es recomendable intercambiar los portales para obtener una visión global de la resección y así evaluar la superficie resecada tanto en pronación como en supinación de forma correcta. Se deberá comprobar que no existen zonas de pinzamiento en la porción más volar y más dorsal. El aspecto más importante a tener en cuenta en ese tipo de resección, es respetar los ligamentos volares ulnocarpianos, la inserción foveal del FCT, la fosa sigmoidea en la porción radial y la inserción de los ligamentos radioulnares volares y dorsales en la porción ulnar, que se encuentran más radiales de lo que se podría esperar. La muñeca debe colocarse en supinación para evitar lesionar la rama sensitiva dorsal del nervio ulnar.

\section{Lesiones Asociadas y Tratamiento}

De forma sistemática y una vez finalizado el procedimiento de wafer artroscópico, se realiza una valoración de la articulación mediocarpiana a través de los portales MCR y MCU, donde se testará la estabilidad del ligamento lunopiramidal y se emplerá la clasificación de Geissler para estadificar el grado de lesión. La lesión del ligamento lunopiramidal está ocasionada por las repetidas transmisiones de fuerzas que pasan a través de la articulación entre el semilunar y el piramidal y por la carga axial transmitida a través de la columna ulnar entre el carpo y la cabeza del cúbito. Para observar su movilidad se empuja el pisiforme contra el piramidal, a nivel volar, mientras se mantiene el piramidal estabilizado. Si se encuentra una lesión asociada del ligamento lunopiramidal se deberá tratar de forma adecuada, aunque normalmente, la lesión del ligamento no es completa, y el desbridamiento con estabilización temporal con agujas de Kirschner (AK) suele dar buenos resultados. ${ }^{7}$ En caso de inestabilidad lunopiramidal grave sin inestabilidad segmentaria volar (VISI), hay autores que aconsejan una osteotomía de acortamiento ulnar, ya que de esa forma, se tensan los ligamentos ulnopiramidales y ulnosemilunares volares y se mejora indirectamente la inestabilidad. ${ }^{8}$

En ocasiones pueden observarse lesiones osteocondrales en el polo proximal del hueso ganchoso, lo que conocemos como el síndrome HALT (Hamate Arthrosis Lunotriquetral Instability). ${ }^{9}$ Esa circunstancia está ligada, frecuentemente, a una variación anatómica del semilunar descrita por Viegas como tipo $2,^{10}$ en la que el semilunar presenta una carilla articular medial que se articula con el polo proximal del ganchoso. En caso de identificar un síndrome HALT asociado durante la exploración del espacio mediocarpiano, 
(-Figura 6) se complementa la resección tipo wafer con una resección del polo proximal del ganchoso de forma artroscópica. ${ }^{11}$ Para ello, se introduce una fresa de 2,9 mm a través del portal MCU con la óptica situada en el portal MCR. Se realiza una resección del polo proximal y radial del ganchoso de unos 2-3 mm utilizando como guía de profundidad el grosor de la fresa. Se debe evitar resecar más de 2-3 mm del polo proximal del ganchoso, por el riesgo de crear una insuficiencia de los ligamentos piramidalganchoso y piramidal-hueso grande.

\section{Manejo Postoperatorio}

Tras un procedimiento tipo wafer, se puede colocar un vendaje blando para un inicio precoz de la movilidad suave de muñeca y el tratamiento de rehabilitación. En caso de encontrar abundante sinovitis o realizar la resección adicional del polo proximal del ganchoso, preferimos inmovilizar la muñeca con una férula antebraquial hasta la retirada de los puntos de sutura (7-10 días) para proporcionar al paciente un postoperatorio inmediato más confortable. Una vez retirada la férula y los puntos de sutura, se coloca una muñequera removible y se inicia el tratamiento rehabilitador. Por el contrario, en caso de desbridamiento y estabilización temporal con agujas de Kirschner del ligamento lunopiramidal, se mantendrá la inmovilización con una férula antebraquial hasta la 5ta semana, momento en que se retirarán las AKy dará comienzo el programa de rehabilitación.

La reincorporación a las tareas deportivas o laborales que requieran esfuerzo tiene lugar entre las 8 y las 12 semanas tras la cirugía.

La queja principal de los pacientes tras la resección tipo wafer es la prolongada duración del dolor articular y la rigidez que parece provenir de la superficie denudada del hueso del cúbito distal expuesta al ambiente articular. Se debe advertir a los pacientes por adelantado, que deberán tolerar eso y que se necesitan entre 4-6 meses para que finalmente desaparezcan esos síntomas.

\section{Tratamiento Rehabilitador}

El tratamiento rehabilitador postquirúrgico deberá ir encaminado a evitar o resolver la posible limitación de supinación que se puede generar y la persistencia de dolor ulnar. Se trabajará la reeducación propioceptiva y se preparará la muñeca para poder desempeñar las actividades cotidianas, laborales y/o deportivas que el paciente desempeñaba previamente.

Habrá que considerar si solo se ha realizado un wafer artroscópico, existía una lesión lunopiramidal añadida, si asociaba lesiones osteocondrales y si además presentaba una inestabilidad radiocubital distal.

La inmovilización postquirúrgica, lo más breve posible, será necesaria para evitar el dolor de carácter irritativo a consecuencia de las acciones quirúrgicas (hay que tener en cuenta que la cobertura de tejidos blandos a ese nivel es escasa y es una superficie de carga). La ortesis deberá colocarse en posición de supinación para descargar las estructuras ulnares durante 7-10 días (se puede dejar nocturna dos semanas más). ${ }^{12}$

Los ejercicios progresarán de posición supinada a neutra y finalmente se realizarán en pronación. La reeducación de la pronación y la prensa no dolorosa será uno de los objetivos primordiales. $^{13}$ Es importante comenzar con las movilizaciones activas precozmente, si es posible aproximadamente a los diez días de la cirugía para evitar la aparición de fibrosis postquirúrgica y una vez haya cedido la clínica dolorosa. Para ello, se podrán utilizar las herramientas farmacológicas disponibles (los corticoides son una muy buena opción a la hora de romper el círculo dolor-edema-inflamación que pueden llevar a ella). Se iniciará el tratamiento con movilizaciones ACTIVAS y AUTOASISTIDAS en flexo-extensión y prono-supinación sin perder de vista el TERT (Total End range time o tiempo total al final del recorrido) y siempre por debajo del dolor. Para trabajar todo el arco de recorrido en supinación será de gran ayuda la MECANOTERAPIA (ej: picas sin y con lastres distales con las que progresivamente se aumentará el brazo de palanca). Si existían lesiones osteocondrales, se asociarán de 10 a 20 sesiones de MAGNETOTERAPIA.

Finalmente, y no antes de las 6 semanas, se introducirán ejercicios de prensa progresiva, siempre teniendo en cuenta la regla del no dolor y con progresión de posición supinada, neutra y finalmente en pronación (la mayor varianza positiva ulnar). Los ejercicios propioceptivos también tendrán una progresión: desde ejercicios en espejo y rodamientos con la soft ball, hasta ejercicios de mayor complejidad de estabilidad dinámica en superficies inestables.

En caso de haber realizado una estabilización lunopiramidal deberemos trabajar específicamente el FCU (Flexor Capi Ulnaris) y el ECU (Extensor Carpi Ulnaris) como estabilizadores secundarios de dicho intervalo. Ante la presencia de una inestabilidad radiocubital distal previa, será el PC (Pronador Cuadrado) y el ECU los músculos que se deberán potenciar más concretamente. ${ }^{14,15}$ Aproximadamente a los 3 meses, el paciente deberá encontrarse preparado para reiniciar su actividad laboral.

\section{Complicaciones}

Las complicaciones descritas para esa técnica quirúrgica son las propias de la artroscopia de muñeca y, por lo tanto, muy escasas. Las complicaciones más características de la técnica son la aparición de una inestabilidad radiocubital distal debido a un fresado excesivo al realizar en procedimiento de wafer y una insuficiencia de los ligamentos piramidal-ganchoso y piramidal-hueso grande si se realiza un fresado excesivo del polo proximal del ganchoso. Las lesiones tendinosas, nerviosas o vasculares son extremadamente raras. Existe riesgo de infección local en el punto de inserción de la AK en caso de inmovilización temporal del ligamento lunopiramidal.

Conflicto de Intereses

Los autores del trabajo declaran no tener ningún conflicto de intereses. 


\section{Bibliografía}

1 Lichtman DM, Alexander AH, Eds. The wrist and its disorders. 2nd ed. Philadelphia, Pa: WB Saunders Co; 1997

2 Harley BJ, Werner FW, Boles SD, Palmer AK. Arthroscopic resection of arthrosis of the proximal hamate: a clinical and biomechanical study. J Hand Surg Am 2004;29(04):661-667

3 Palmer AK. Triangular fibrocartilage complex lesions: a classification. J Hand Surg Am 1989;14(04):594-606

4 Sammer DM, Rizzo M. Ulnar impaction. Hand Clin 2010;26(04): 549-557

5 Lucas FJ, Carratalá V, Sanchez E. Generalidades, portales y anatomía artroscópica. Rev Esp Artrosc Cir Articul 2014;21:5-13

6 Nakamura T, Yabe Y, Horivachi Y. Functional anatomy of the triangular fibrocatilage complex.J Hand Surg Am 1996;21B:581-586

7 Osterman AL, Seidman GD. The role of arthroscopy in the treatment of lunatotriquetral ligament injuries. Hand Clin 1995;11(01):41-50

8 Tiedeken NC, Baratz ME. Ulnocarpal Impingement and Triangular Fibrocartilage Complex Tears. Oper Tech Sports Med 2016;24 (02):131-138
9 Harley BJ, Werner FW, Boles SD, Palmer AK. Arthroscopic resection of arthrosis of the proximal hamate: a clinical and biomechanical study. J Hand Surg Am 2004;29(04):661-667

10 Viegas SF, Wagner K, Patterson R, Peterson P. Medial (hamate) facet of the lunate. J Hand Surg Am 1990;15(04):564-571

11 Carratala V, Lucas F, Sánchez E. Dolor en el borde ulnar de la muñeca. Rev esp artrosc cir articul 2014;21:77-80

12 Michael Lee et al. Ulnar wrist pain and impairment: a therapist's algorithmic approach to the triangular fibrocatilage complex. Rehabilitation of the hand and upper extremity. 6th Ed ed. Mosby/ Elsevier; 2016:974-981

13 Heinekamp Jochen-Frederick et al. Long-term functional outcome and patient satisfaction after ulnar resection. JPlast Reconstr Aesthet Surg 2016;69(10):1417-1423

14 Lluch A, Salva G, Esplugas M, Hagert E, Garcia Elias M. El papel de la propiocepción y el control neuromuscular en las inestabilidades del carpo. Rev Ibroarm Cir mano 2015;43:70-78

15 Guisasola E, Carratala V, Calduch F, Lucas F. El papel de la rehabilitación tras las reparaciones de las inestabilidades de muñeca. Rev Iberoam Cir mano 2016;44(02):131-144 